OPEN ACCESS

Edited by:

Massimo Imazio

University Hospital of the City of Health and Science of Turin, Italy

Reviewed by:

Irma Bisceglia,

San Camillo-Forlanini Hospital, Italy

Valentina Sala,

University of Turin, Italy

*Correspondence: Jun-ichi Abe

jabe@mdanderson.org

Joerg Herrmann

herrmann.joerg@mayo.edu

Specialty section:

This article was submitted to

Cardio-Oncology,

a section of the journal

Frontiers in Cardiovascular Medicine

Received: 01 September 2020

Accepted: 26 October 2020

Published: 25 November 2020

Citation:

Abe J-i, Yusuf SW, Deswal A and Herrmann J (2020) Cardio-Oncology: Learning From the Old, Applying to the New.

Front. Cardiovasc. Med. 7:601893.

doi: $10.3389 / f c v m .2020 .601893$

\section{Cardio-Oncology: Learning From the Old, Applying to the New}

\author{
Jun-ichi Abe ${ }^{1 *}$, Syed Wamique Yusuf ${ }^{1}$, Anita Deswal ${ }^{1}$ and Joerg Herrmann ${ }^{2 *}$ \\ ${ }^{1}$ Department of Cardiology, University of Texas MD Anderson Cancer Center, Houston, TX, United States, ${ }^{2}$ Department of \\ Cardiovascular Medicine, Mayo Clinic, Rochester, MN, United States
}

The recent surge in cancer drug approval has provided us in cardio-oncology with a new and unique era, which modern medicine has not experienced before: the diminishing availability of "conventional" evidence-based medicine. The drastic and quick changes in oncology has made it difficult, and at times even impossible, to establish a meaningful evidence-based cardio-oncology practice by simply following the oncologists' practice. For the modern cardio-oncologist, it seems that a more proactive approach and methodology is needed. We believe that only through such an approach (learn from the old, and apply to the new) the cardio-oncologist will obtain meaningful evidence to perform their every-day practice in this new era.

Keywords: evidence-based medicine, type 1 and type 2 cardiotoxicity, anthracycline, trastuzumab, onco-cardiology, cardio-oncology

\section{INTRODUCTION}

Over the last few years, the field of cardio-oncology has seen a number of new developments, challenges, and changes in paradigms. First, with advancements in cancer therapy, long-term survival has reached an all-time high. In 2016, in the U.S. alone, the cancer survivor population increased to over 15 million with a projection to over 26 million by 2040 . Of further interest, nearly three quarters of these patients are 65 years and older with a higher burden of comorbidities, most frequently cardiovascular in nature $(1,2)$. Cancer and cardiovascular disease (CVD) notably share risk factors, for example, aging, smoking, and obesity. Also, not only has cancer and its treatment been linked to CVD, but certain CVD conditions, such as heart failure and myocardial infarction, have been linked to an increased risk of cancer as well (3-7). Thus, there is an undeniable and unavoidable intersection of cancer and CVD. While basic and translational research has delineated some of the pathophysiological details in this area, much is still unknown (research lag and gap) and a number of best practice aspects are not defined (practice and education lag and gap).

\section{WHERE DO WE COME FROM? CHANGING PARADIGMS}

Historically, much in the field of cardiotoxicity has focused on anthracyclines and trastuzumab. This did provide important insight into mechanisms and principles of how cancer treatments can negatively affect cardiac function. Ewer and Lippman introduced the concept of type 1, irreversible, and type 2, reversible, cardiotoxicity (8). The post-erchild for the first type are anthracyclines such as doxorubicin, which cause dose-dependent injury to the myocardium by many different mechanisms, one of the most important being induction of oxidative stress with related modifications of lipid, protein, and genetic molecules $(9,10)$. Anthracyclines show high affinity for metal ions, and reduction of the quinone moiety in complex with $\mathrm{Fe}^{+}$may accelerate 
reactive oxygen species (ROS) formation (Fenton reaction) (11). Another mechanism is the interaction of anthracyclines with topoisomerase $2 \beta$ (TOP2 $\beta$ ). Anthracyclines bind to both, DNA and TOP2, to form the DNA cleavage complex, which blocks DNA replication, inducing DNA double strand breaks and cell death. TOP $2 \alpha$ is highly expressed in proliferating cells such as cancer cells and accounts for the therapeutic action. On the contrary, TOP $2 \beta$ is expressed in the myocardium and leads to the cardiotoxic action (12).

In distinction to the above, initial experience with trastuzumab outlined the occurrence of a different type of cardiac dysfunction (type 2) that was dose-independent and mostly reversible (13-17). Trastuzumab targets one of the outer domains of the HER-2/Erb2receptor, which is overexpressed in $\sim 20-25 \%$ of breast cancers and confers a poor prognosis, thus granting pivotal significance to directed therapies $(13,17)$. Cardiotoxicity, however, remains one of the most important side effects that can be therapy-limiting and therapy-terminating. Intriguingly, this side effect was not anticipated as the role of HER-2/Erb2 signaling in the myocardium was not known at the time. In fact, it was the introduction of trastuzumab that has led to the recognition of HER-2/Erb2 signaling as a myocardial stress response pathway, which is activated and crucial under high afterload conditions and anthracycline exposure (18).

Recently, arguments against the type 1 vs. type 2 concept have arisen, in view of the fact that doxorubicin-induced cardiotoxicity is not always irreversible $(9,10)$, and on the other hand, trastuzumab-induced cardiotoxicity is not always reversible (19). Indeed, it might be more a matter of the type, timing, duration, and combination of drugs given within the broader context of the patient's genetic and comorbidity profile that determines the trajectory of cardiotoxicity. Indeed, overlap is not inconceivable, and in fact, the sequential and concurrent stress of anthracyclines and trastuzumab has been welldocumented. In this context it should be noted that the effects of anthracyclines and trastuzumab on vascular components may be underestimated (20). Doxorubicin can impact endothelial cells, vascular smooth muscle cells, and monocyte/macrophages (20-22). Likewise, trastuzumab treatment can induce a M1like macrophage phenotype (23) and endothelial dysfunction (24). These vascular aspects may contribute to and complicate the interpretation of cardiotoxicity due to anthracyclines or trastuzumab $(25,26)$.

For the reasons outlined, any subtyping of cardiotoxicity is inherently difficult. While one may argue about some overlap between the type 1 and type 2 cardio-toxicities (27), the concept did, however, help move the field forward, and developing and testing principles and theories will remain key in the emerging discipline of Cardio-Oncology [Figure 1; (28)].

A number of other intertwined molecular processes induced by various cancer treatments, including mitochondrial-related defects [e.g., iron accumulation (11, 29), impaired biogenesis and clearance (30)], degradation of myofilaments (31), and alteration of survival pathways (32), have been suggested, and are discussed extensively in other reviews $(25,33,34)$. These multifactorial process and mechanisms, leading to cardiovascular disease in cancer survivors, complicate further the understanding of the pathophysiology of various cancer treatment-induced cardiovascular toxicities, and make it difficult to translate those basic findings into an optimized clinical practice.

\section{WHO ARE WE? CHANGING TIMES}

Cancer drugs account for $27 \%$ of all new drugs in the United States since 2010, with the FDA having approved a total of 126 cancer drugs to treat solid and hematologic tumors from 1980 through 2018 (https://ascopost.com/news/ september-2019/cancer-drugs-account-for-over-a-quarter-ofall-new-drug-approvals-in-the-us/). In view of this surge of new therapies, the recent practice of oncology has been changing very frequently and dramatically. One of the most impressive revolutionary milestones in this regard is the development of immune checkpoint inhibitors (ICIs). Currently, hundreds of phase I, II, and phase III/IV clinical trials of anti-PD-1, PD-L1, and CTLA-4 are being carried out across the globe (35). Another ground-breaking development in the area of immuno-oncology has been that of chimeric antigen receptor (CAR) T-cell [and biphasic $\mathrm{T}$ cell engager (BITE)] therapy. Furthermore, many chemical inhibitors targeting key DNA damage response proteins, including DNA-PKs (DNA-dependent protein kinase, catalytic subunit), ATM/ATR (ataxia-telangiectasia mutated and Rad3-related), the MRN (MRE11-RAD50-NBS1) complex, and the PARP (poly(ADP-ribose) polymerase) family are emerging as promising cancer therapies (36). On a flip side, the utilization of classical chemotherapeutics or their doses is now diminishing, e.g., anthracycline use in breast cancer.

While these developments illustrate advancements with the goal of improved outcomes, they also point to new challenges. In particular, the scope of oncology is so broad and specialized at the same time with newly emerging drugs and changing practice patterns that no time is given to cardio-oncologists to adapt and generate the evidence necessary for the best management or even prevention of cardiovascular toxicities. For example, if a particular cancer drug is no longer used by the time a cardio-oncologist can define its cardiovascular toxicity profile, management, and prevention approach, those discoveries and insights will be less meaningful. The oncologist will not wait for us (Figure 2).

This being said, some cardio-oncology practice aspects will remain strong, grounded in the general principles of cardiovascular medicine. For example, the management of preexisting CVD will always have an important role in preventing cardiovascular events. Along those lines, cardio-oncologists are relying on the knowledge of general cardiology and internal medicine. However, general CV principles and treatment of comorbidities will not be enough, and it is necessary to add specifics that are nuanced for the particular agents. Due to the rapid changes and the shortage of time, the current cardioonclogists are forced to practice without knowing the specificities and without adequate evidence, using a form of "Band-Aid" practice (Figure 2). 


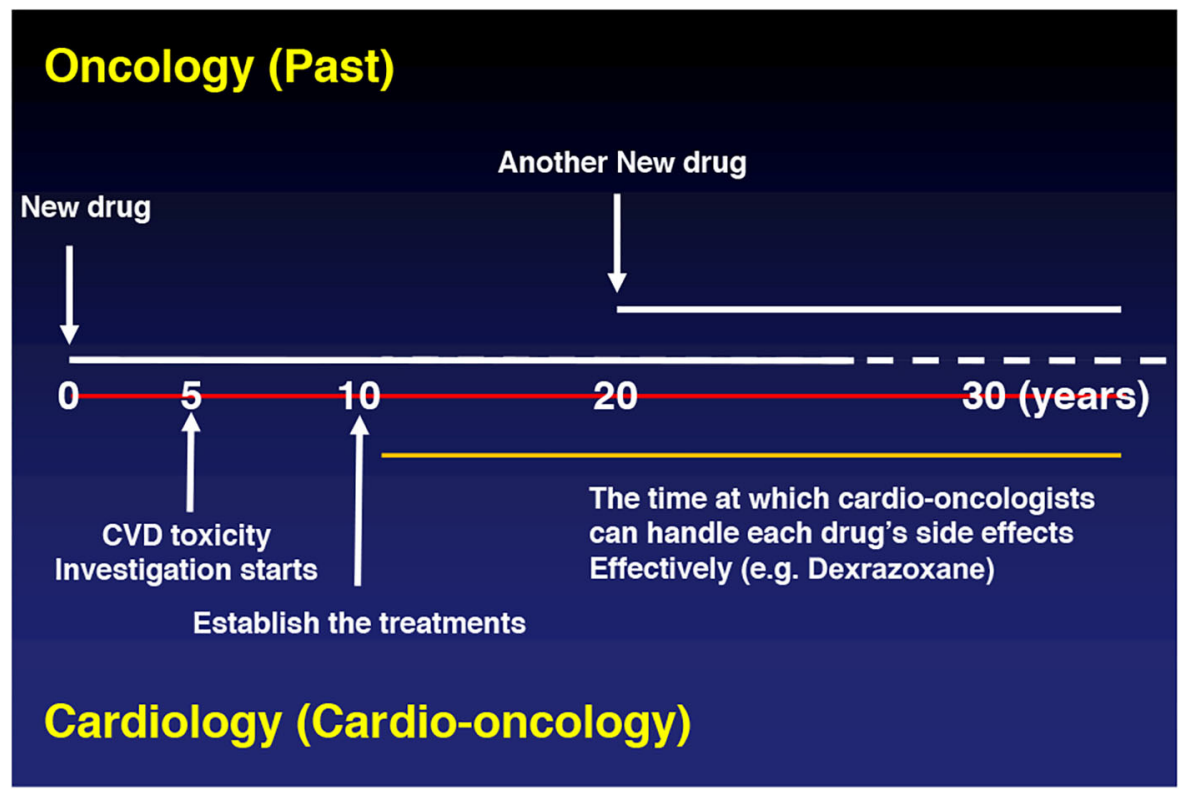

FIGURE 1 | Past.

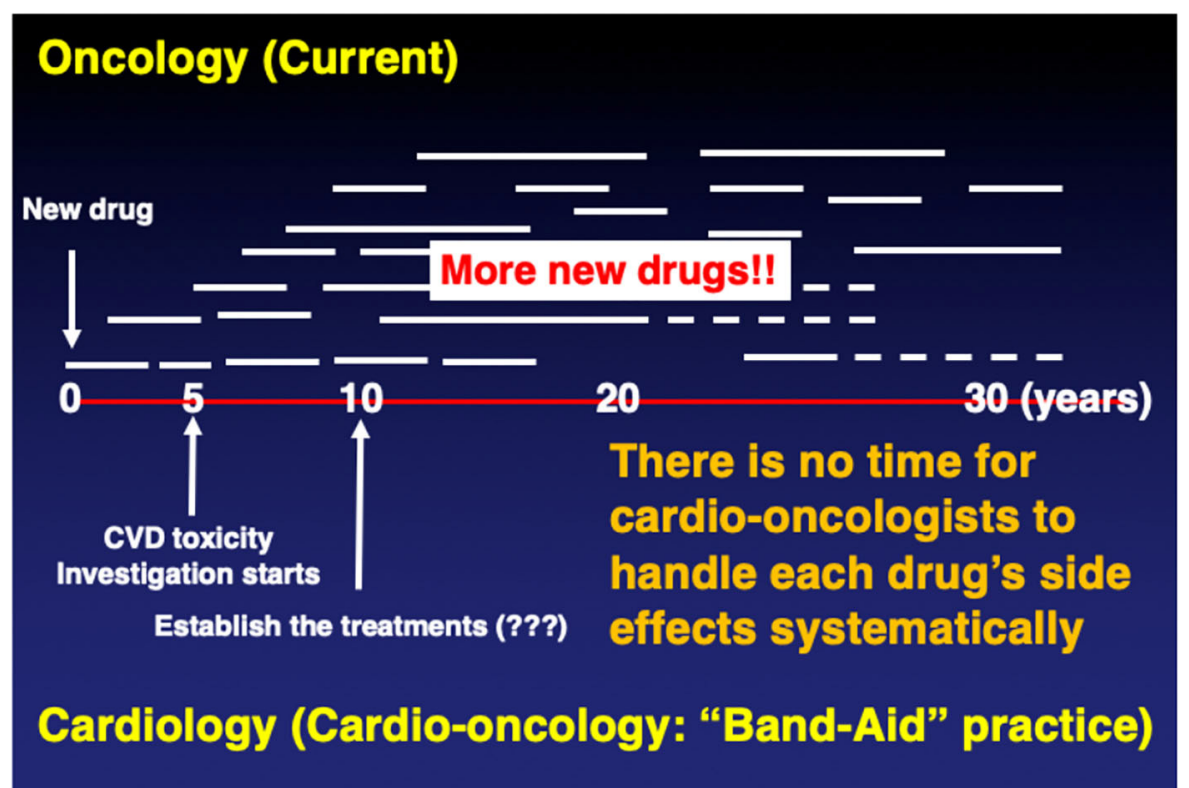

FIGURE 2 | Current.

\section{WHERE ARE WE GOING? CHANGING MINDS}

In view of the outlined developments there is a need to prepare cardio-oncology specialists for the current and future developments. A key need is structured training in cardio-oncology with core knowledge and core competency requirements. The cardiology fellowship should have a dedicated curriculum for Cardio-Oncology so that even the general cardiologist can have a better understanding of cancer patients afflicted with cardiovascular diseases. Cardiologists subspecializing in this field should spend at least 6-12 months of clinical training in this field, followed by a subspecialty examination. An initial outline of a cardio-oncology training program has been drafted by the American College of Cardiology, and a board-style certification examinations has been launched 


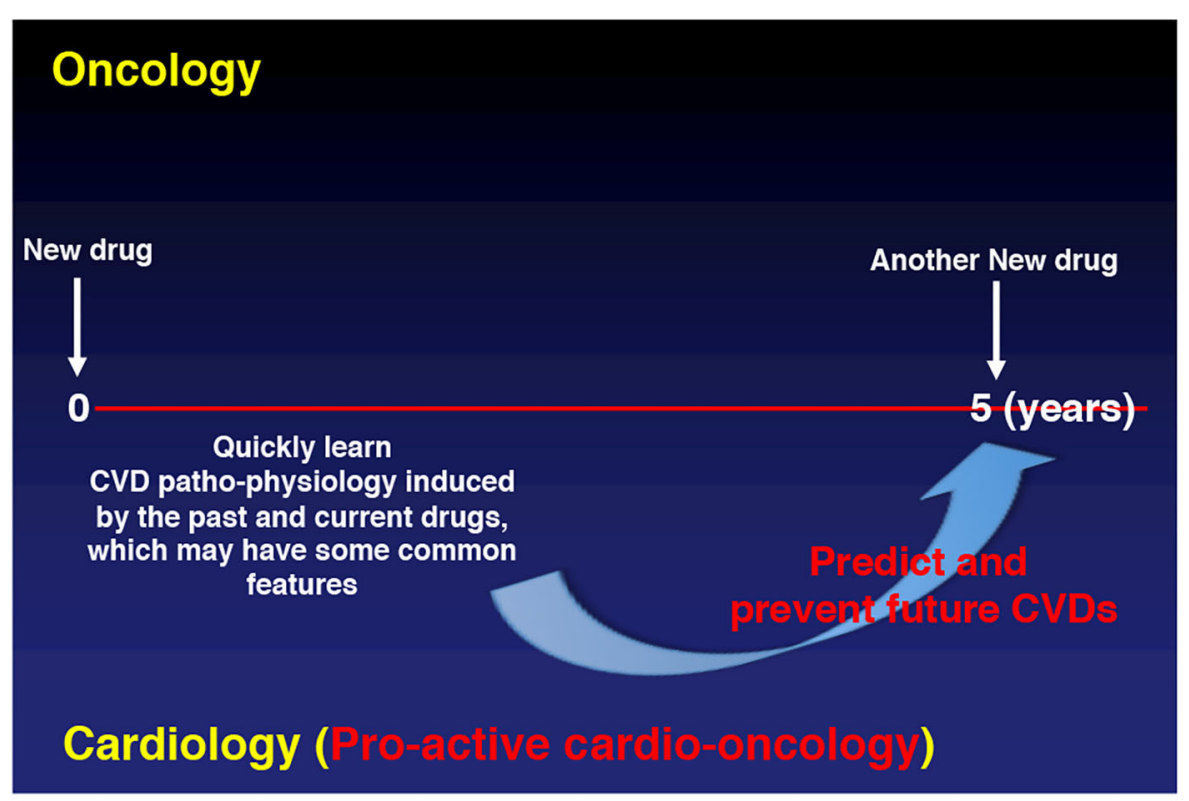

FIGURE 3 | Future.

by the International Cardio-Oncology Society (ICOS). Other societies and regulatory institutions should follow with the goal of providing nationally and even universally accepted credentials (e.g., the American College of Cardiology and the American Board of Internal Medicine). Aligned with these efforts is the need for standardization of the definition of cardiovascular toxicities in a joint, cross-disciplinary manner. Such initiatives will help clinical practice as well as research, allowing for the direct comparison of outcomes. Furthermore, there should be standardization not only in terms of outcomes but also in terms of availability of cardio-oncology services. Quality and outcomes are another aspect that will be key to further substantiate the importance of the field.

Providing the evidence base for these practice and education related efforts is of utmost importance. It calls for research that will provide new insight, mechanistically, translationally, and clinically, and help transition cardio-oncology from a "BandAid" to an evidence-based practice approach. These research efforts should cover the entire continuum of cancer care, from before, to the during, and the after cancer therapy with most

\section{REFERENCES}

1. Coebergh JW, Janssen-Heijnen ML, Post PN, Razenberg PP. Serious co-morbidity among unselected cancer patients newly diagnosed in the southeastern part of The Netherlands in 1993-1996. J Clin Epidemiol. (1999) 52:1131-6. doi: 10.1016/S0895-4356(99)00 098-0

2. Bluethmann SM, Mariotto AB, Rowland JH. Anticipating the "Silver Tsunami": prevalence trajectories and comorbidity burden among older cancer survivors in the United States. Cancer Epidemiol Biomarkers Prev. (2016) 25:1029-36. doi: 10.1158/1055-9965.EPI-16-0133 optimal prevention, surveillance, and treatment. Furthermore, at the time of drug development itself, its potential for cardiovascular toxicity should be carefully evaluated such that at the time of its introduction one can be prepared to expect and address emerging issues. Big data and artificial intelligence may aid in this regard and steer into a bright future. As outlined herein, what is needed are more proactive approaches to change the cardio-oncology practice from "dealing with the common final CV adverse effects" by merely following the oncology practice, to an evidence-based practice by learning the pathophysiology and principles of cardiovascular toxicities across cancer treatments (Figure 3).

Changing paradigms, changing times, and changing minds, this is how we see the past, present, and future of cardio-oncology.

\section{AUTHOR CONTRIBUTIONS}

All authors listed have made a substantial, direct and intellectual contribution to the work, and approved it for publication. 
targeted therapy and immunotherapy. Curr Opin Cardiol. (2019) 34:441-50. doi: 10.1097/HCO.0000000000000641

7. Koelwyn GJ, Newman AAC, Afonso MS, van Solingen C, Corr EM, Brown EJ, et al. Myocardial infarction accelerates breast cancer via innate immune reprogramming. Nat Med. (2020) 26:1452-8. doi: 10.1038/s41591-020-0964-7

8. Ewer MS, Lippman SM. Type II chemotherapy-related cardiac dysfunction: time to recognize a new entity. J Clin Oncol. (2005) 23:2900-2. doi: 10.1200/JCO.2005.05.827

9. Cardinale D, Colombo A, Lamantia G, Colombo N, Civelli M, De Giacomi $\mathrm{G}$, et al. Anthracycline-induced cardiomyopathy: clinical relevance and response to pharmacologic therapy. J Am Coll Cardiol. (2010) 55:21320. doi: 10.1016/j.jacc.2009.03.095

10. Cardinale D, Colombo A, Bacchiani G, Tedeschi I, Meroni CA, Veglia F, et al. Early detection of anthracycline cardiotoxicity and improvement with heart failure therapy. Circulation. (2015) 131:1981-8. doi: 10.1161/CIRCULATIONAHA.114.013777

11. Gammella E, Maccarinelli F, Buratti P, Recalcati S, Cairo G. The role of iron in anthracycline cardiotoxicity. Front Pharmacol. (2014) 5:25. doi: $10.3389 /$ fphar.2014.00025

12. Zhang S, Liu X, Bawa-Khalfe T, Lu LS, Lyu YL, Liu LF, et al. Identification of the molecular basis of doxorubicin-induced cardiotoxicity. Nat Med. (2012) 18:1639-42. doi: 10.1038/nm.2919

13. Slamon DJ, Leyland-Jones B, Shak S, Fuchs H, Paton V, Bajamonde A, et al. Use of chemotherapy plus a monoclonal antibody against HER2 for metastatic breast cancer that overexpresses HER2. N Engl J Med. (2001) 344:783-92. doi: 10.1056/NEJM200103153441101

14. Suter TM, Cook-Bruns N, Barton C. Cardiotoxicity associated with trastuzumab (Herceptin) therapy in the treatment of metastatic breast cancer. Breast. (2004) 13:173-83. doi: 10.1016/j.breast.2003.09.002

15. Tripathy D, Seidman A, Keefe D, Hudis C, Paton V, Lieberman G. Effect of cardiac dysfunction on treatment outcomes in women receiving trastuzumab for HER2-overexpressing metastatic breast cancer. Clin Breast Cancer. (2004) 5:293-8. doi: 10.3816/CBC.2004.n.033

16. Guarneri V, Lenihan DJ, Valero V, Durand JB, Broglio K, Hess KR, et al. Long-term cardiac tolerability of trastuzumab in metastatic breast cancer: the M.D. Anderson Cancer Center experience. J Clin Oncol. (2006) 24:410715. doi: 10.1200/JCO.2005.04.9551

17. Romond EH, Jeong JH, Rastogi P, Swain SM, Geyer CEJr, Ewer MS, et al. Seven-year follow-up assessment of cardiac function in NSABP B-31, a randomized trial comparing doxorubicin and cyclophosphamide followed by paclitaxel (ACP) with ACP plus trastuzumab as adjuvant therapy for patients with node-positive, human epidermal growth factor receptor 2-positive breast cancer. J Clin Oncol. (2012) 30:3792-9. doi: 10.1200/JCO.2011.40.0010

18. Chen B, Peng X, Pentassuglia L, Lim CC, Sawyer DB. Molecular and cellular mechanisms of anthracycline cardiotoxicity. Cardiovasc Toxicol. (2007) 7:114-21. doi: 10.1007/s12012-007-0005-5

19. De Azambuja E, Procter MJ, Van Veldhuisen DJ, Agbor-Tarh D, MetzgerFilho O, Steinseifer J, et al. Trastuzumab-associated cardiac events at 8 years of median follow-up in the Herceptin Adjuvant trial (BIG 1-01). J Clin Oncol. (2014) 32:2159-65. doi: 10.1200/JCO.2013.53.9288

20. Herrmann J. Vascular toxic effects of cancer therapies. Nat Rev Cardiol. (2020) 17:503-22. doi: 10.1038/s41569-020-0347-2

21. Kotamraju S, Konorev EA, Joseph J, Kalyanaraman B. Doxorubicin-induced apoptosis in endothelial cells and cardiomyocytes is ameliorated by nitrone spin traps and ebselen. Role of reactive oxygen and nitrogen species. J Biol Chem. (2000) 275:33585-92. doi: 10.1074/jbc.M003890200

22. He H, Wang L, Qiao Y, Zhou Q, Li H, Chen S, et al. Doxorubicin induces endotheliotoxicity and mitochondrial dysfunction via ROS/eNOS/NO pathway. Front Pharmacol. (2019) 10:1531. doi: 10.3389/fphar.2019.01531
23. Bloom MJ, Jarrett AM, Triplett TA, Syed AK, Davis T, Yankeelov TE, et al. Anti-HER2 induced myeloid cell alterations correspond with increasing vascular maturation in a murine model of HER2+ breast cancer. BMC Cancer. (2020) 20:359. doi: 10.1186/s12885-020-06868-4

24. Sandoo A, Kitas GD, Carmichael AR. Endothelial dysfunction as a determinant of trastuzumab-mediated cardiotoxicity in patients with breast cancer. Anticancer Res. (2014) 34:1147-51.

25. Herrmann J, Yang EH, Iliescu CA, Cilingiroglu M, Charitakis $\mathrm{K}$, Hakeem A, et al. Vascular toxicities of cancer therapies: the old and the new-an evolving avenue. Circulation. (2016) 133:1272-89. doi: 10.1161/CIRCULATIONAHA.115.018347

26. Ma X, Zhu P, Ding Y, Zhang H, Qiu Q, Dvornikov AV, et al. Retinoid $\mathrm{X}$ receptor alpha is a spatiotemporally predominant therapeutic target for anthracycline-induced cardiotoxicity. Sci Adv. (2020) 6:eaay2939. doi: 10.1126/sciadv.aay2939

27. Ewer MS, Ewer SM. Trastuzumab cardiotoxiciy: the age-old balance of risk and benefit. Br J Cancer. (2016) 115:1441-2. doi: 10.1038/bjc.2016.381

28. Herrmann J. Adverse cardiac effects of cancer therapies: cardiotoxicity and arrhythmia. Nat Rev Cardiol. (2020) 17:474-502. doi: 10.1038/s41569-020-0348-1

29. Fojtu M, Gumulec J, Stracina T, Raudenska M, Skotakova A, Vaculovicova $\mathrm{M}$, et al. Reduction of doxorubicin-induced cardiotoxicity using nanocarriers: a review. Curr Drug Metab. (2017) 18:237-63. doi: 10.2174/1389200218666170105165444

30. Bartlett JJ, Trivedi PC, Pulinilkunnil T. Autophagic dysregulation in doxorubicin cardiomyopathy. J Mol Cell Cardiol. (2017) 104:1-8. doi: 10.1016/j.yjmcc.2017.01.007

31. Hantson P. Mechanisms of toxic cardiomyopathy. Clin Toxicol. (2019) 57:19. doi: $10.1080 / 15563650.2018 .1497172$

32. Iqubal A, Iqubal MK, Sharma S, Ansari MA, Najmi AK, Ali SM, et al. Molecular mechanism involved in cyclophosphamideinduced cardiotoxicity: old drug with a new vision. Life Sci. (2019) 218:112-31. doi: 10.1016/j.lfs.2018.12.018

33. Yeh ET, Bickford CL. Cardiovascular complications of cancer therapy: incidence, pathogenesis, diagnosis, and management. J Am Coll Cardiol. (2009) 53:2231-47. doi: 10.1016/j.jacc.2009.02.050

34. Jain D, Russell RR, Schwartz RG, Panjrath GS, Aronow W. Cardiac complications of cancer therapy: pathophysiology, identification, prevention, treatment, and future directions. Curr Cardiol Rep. (2017) 19:36. doi: 10.1007/s11886-017-0 $846-\mathrm{x}$

35. Tang J, Yu JX, Hubbard-Lucey VM, Neftelinov ST, Hodge JP, Lin Y. Trial watch: The clinical trial landscape for PD1/PDL1 immune checkpoint inhibitors. Nat Rev Drug Discov. (2018) 17:854-5. doi: 10.1038/nrd.201 8.210

36. O'Connor MJ. Targeting the DNA damage response in cancer. Mol Cell. (2015) 60:547-60. doi: 10.1016/j.molcel.2015.10.040

Conflict of Interest: The authors declare that the research was conducted in the absence of any commercial or financial relationships that could be construed as a potential conflict of interest.

Copyright (๑) 2020 Abe, Yusuf, Deswal and Herrmann. This is an open-access article distributed under the terms of the Creative Commons Attribution License (CC BY). The use, distribution or reproduction in other forums is permitted, provided the original author(s) and the copyright owner(s) are credited and that the original publication in this journal is cited, in accordance with accepted academic practice. No use, distribution or reproduction is permitted which does not comply with these terms. 